\title{
Two-dimensional imaging of neutral alkali atom samples using surface ionization
}

\author{
Katharina Christandl, Gregory P. Lafyatis, ${ }^{\text {a) }}$ Andrei Modoran, and Tung-Hsiu Shih \\ Department of Physics, The Ohio State University, Columbus, Ohio 43210
}

(Received 25 June 2002; accepted 9 September 2002)

\begin{abstract}
We describe the design and characterization of a high resolution, high efficiency detector for two-dimensional imaging of neutral atoms. Incident atoms are surface-ionized by a tungsten-coated hot ribbon and the resulting ions are accelerated into a microchannel plate detector with a phosphor screen output. With this design we can detect individual alkali and other low ionization potential atoms and molecules with a spatial resolution of $\sim 20 \mu \mathrm{m}$. We find backgrounds on the order of 30 $\mathrm{Hz}$ over an active detection region of $20 \mathrm{~mm}^{2}$, a time response of $\leqslant 1 / 30 \mathrm{~s}$, and a detection efficiency of $\sim 50 \%$. This detector can be used to image cold as well as thermal atomic or molecular beams. (C) 2002 American Institute of Physics. [DOI: 10.1063/1.1518149]
\end{abstract}

\section{INTRODUCTION}

The recent rapid increase in experimental research using ultracold neutral atom samples motivates the development of methods to detect neutral atomic species. Frequently the design or even the feasibility of an experiment is determined by the detection options that are available. An example of this is the case of certain cold atom experiments that use metastable noble gas species. ${ }^{1,2}$ From a purely technical perspective, it is much easier to make ultracold alkali samples than ultracold metastable noble gas samples. Despite this, some cold atom experiments use the latter, largely because it is straightforward to image metastable samples with both high spatial resolution and high detection efficiency. Because the metastable detection scheme shares much with our own, we outline it here. The metastable detector itself consists of a microchannel plate (MCP) with a phosphor screen at its output. The metastable sample to be imaged is directed toward the $\mathrm{MCP}$; in the case of cold atoms, often the sample is simply dropped onto the MCP. Now usually, when a metastable noble gas atom hits the MCP surface, it will give up its electronic energy by kicking an electron out of the surface and this electron will initiate an electron avalanche pulse in the MCP. In this way, an image of the metastable sample hitting the MCP is created on the phosphor screen. For quantitative work the phosphor screen may be monitored by a television camera feeding a frame grabber. Metastables are detected with order-unity efficiency and a spatial resolution limited only by the pore spacing of the MCP, $\sim 10-15 \mu \mathrm{m}$. We have developed a detector with similar characteristics for neutral alkali atomic and molecular species.

Most ultracold atom experiments use alkali species and for these the most widely used detection options are either a Langmuir-Taylor "hot wire" detector based on the surface ionization process or a laser induced fluorescence arrangement. Some detection considerations were recently discussed by Delhuille et al. $^{3}$ Laser induced fluorescence allows for

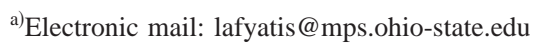

imaging; however, stray light makes it difficult to detect small numbers of atoms. Also, the detection efficiency decreases rapidly as the velocity of the incident atoms increases, which renders this approach unsuitable for analyzing extremely weak thermal atom beams. Surface ionization detectors were originally developed by Taylor ${ }^{4}$ based on the work of Langmuir and Kingdon. ${ }^{5}$ A detector usually consists of a heated thin wire or ribbon of tungsten or some other metal with a high work function. Neutral atoms or molecules that strike the wire can boil off as positive ions and these may be either measured as a current or detected, individually, using an electron multiplier and particle counting electronics. One-dimensional (1D) spatial information, for example, a 1D transverse profile of a beam, can be obtained by systematically moving the detector around. But, this is time consuming and may not be practical for experiments that require measuring very small numbers of atoms with high spatial resolution.

The basic idea of our detector is to direct the neutral alkali atom-in this work, $\mathrm{Rb}$ - sample that we wish to image towards a heated metal surface and allow surface ionization to create positive ions. These are accelerated a short distance into an MCP to initiate electron avalanches, and, in a fashion identical to the noble gas metastable detector, at the MCP output is a phosphor screen that is monitored by a television camera. The result is a detector with high spatial resolution and high detection efficiency that can detect alkali atoms or any other atomic or molecular species with a low ionization potential. Specifically, in this article we describe a device that detects individual alkali atoms, with spatial resolution $\sim 20 \mu \mathrm{m}$, detection efficiency $\sim 50 \%$, and has a background count rate on the order of $30 \mathrm{~Hz}$ over a sensitive area of about $20 \mathrm{~mm}^{2}$. It can be used for detection of cold as well as thermal atoms.

There were two major technical obstacles in developing the detector. First, we tried several candidate metal samples for the "heated metal surface" and found that for samples of the highest purity that is commercially available, the trace 


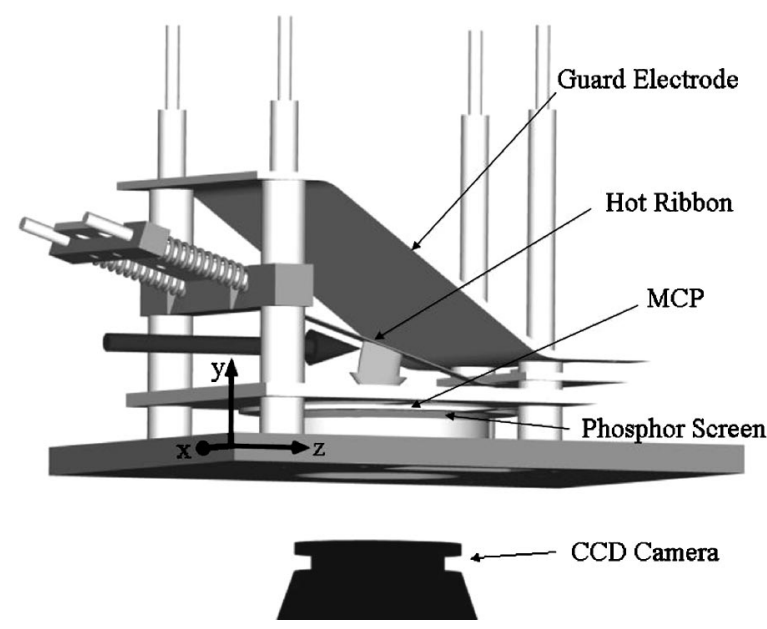

FIG. 1. The detector. Neutral atoms (dark arrow) are incident on a $1000 \mathrm{~K}$ foil ribbon and are ionized by the surface. The ions (wide, light gray arrow) are accelerated through a potential of $\sim 4 \mathrm{kV}$ onto a channel plate electron multiplier. The output of the electron multiplier is directed to a phosphor screen that is monitored by a television camera. The ions follow the electric field lines, which, for this geometry, are simply circular arcs centered on the axis of the cylinder segment made up of the ribbon and the MCP. The incident atomic beam travels in the $z$ direction. The $18^{\circ}$ tilt of the ribbon with respect to the incident atomic beam results in an increase of a factor of 3.2 in spatial resolution along the $y$ direction. Not shown in the figure is a cooled copper housing that surrounds most of the detector during operation.

amounts of alkali atoms in the metal itself would also surface ionize and cause enormous backgrounds (i.e., hundreds of $\mathrm{kHz}$ ). To make a useful detector, these backgrounds had to be reduced to tens of $\mathrm{Hz}$. Second, to have good spatial resolution, it is desirable to get the hot metal surface close to the $\mathrm{MCP}$, in our detector the spacing is $\lesssim 1 \mathrm{~cm}$. But, at the same time, the heat radiating to the MCP is potentially destructive to that device and, as we discuss below, can cause other problems.

Sheehy et al. ${ }^{6}$ describe a detector that is in many ways similar to ours. They located at $70 \%$ transmission, heated nickel grid directly in front of a MCP detector. Like us, they displayed the MCP output on a phosphor screen that was monitored by a television camera. In operation, alkali atoms that hit the nickel grid usually boiled off as ions. The biasing was such that a fraction of these ions would be accelerated on through the grid and onto the MCP surface where they were detected, ultimately by the television system. Their arrangement allowed them to make $2 \mathrm{D}$ spatial measurements of the transverse distributions of atoms in atomic beams. They quote a spatial resolution $\sim 500 \mu \mathrm{m}$ but make no mention of detecting individual atomic particles.

\section{DESIGN}

Figure 1 shows the overall design of the detector. Neutral $\mathrm{Rb}$ atoms hit a $20 \mathrm{~mm}$ long by $3 \mathrm{~mm}$ wide heated, tungsten-coated, rhenium foil ribbon. Atoms usually boil off the surface of the ribbon as (singly charged) ions. The ribbon is typically biased about $4 \mathrm{kV}$ above the MCP input, which is held at ground potential. The ribbon and MCP form an $18^{\circ}$ pie-shaped segment of a cylinder. Ions travel to the MCP very nearly along the electric field lines, which, for this geometry, are just circular arcs centered on the cylinder axis.
The MCP that we use has $10 \mu \mathrm{m}$ holes spaced on a $12 \mu \mathrm{m}$ pattern. A third, "guard" electrode is located as shown in Fig. 1 and biased so as to reduce fringing fields around the ribbon.

Our envisioned applications involve looking for DeBroglie wave interference fringes in cold beams of atoms that have passed through various thin slits or transmission gratings. Here, all atoms travel very nearly in the same direction, the beam axis or $z$ in Fig. 1, and the detector gives the distribution of atoms in the plane transverse to that direction. In addition, it is the resolution along the direction perpendicular to the slits and grating lines that matters most to us and we arrange this to be the " $y$ " direction in the figure. The tilt of the hot ribbon relative to the incident beam is such that atoms separated by $\delta y$ in the $y$ direction land on the hot ribbon and in turn the MCP separated by $\delta y / \sin 18^{\circ}$, and this effectively improves the resolution of the detector in that direction by a factor of $1 / \sin 18^{\circ} \simeq 3.2$. When we quote a resolution of $20 \mu \mathrm{m}$, it refers to the $y$ direction only. The spatial resolution for the $x$ direction or randomly directed atoms is $50-60 \mu \mathrm{m}$.

A chevron configured pair of MCP electron multipliers is employed for the ions to generate pulses of electrons that are in turn accelerated onto a phosphor screen. The gap between the MCP output and the phosphor screen is $0.5 \mathrm{~mm}$ and the accelerating voltage between the two is typically about 1350 V. We chose a phosphor type P22 for the screen because it has high conversion efficiency and is reasonably fast. The screen is viewed by a charge coupled device (CCD) television camera whose output is directed either to a monitor or a frame grabber board in a computer. Image processing was done using LabVIEW software. Spots in the image were identified and the center of each spot was found. These correspond to the locations of individual $\mathrm{Rb}$ atoms in the sample as they hit the ribbon.

The heart of the detector is the hot ribbon. It was cut from a $12.7 \mu \mathrm{m}$ thick rhenium foil using an electric discharge machine in order to minimize arcing problems due to sharp edges. Preliminary measurements with the bare foil yielded backgrounds of at least hundreds of kilohertz due to trace alkali impurities. These were reduced to tolerable levels as follows. First, a $2 \mu \mathrm{m}$ thick tungsten coating was applied to the ribbon based on a procedure originally described by Frazer et al.: ${ }^{7}$ In a separate vacuum chamber the ribbon was heated to $\sim 1400 \mathrm{~K}$ (yellow hot) in a $\mathrm{W}(\mathrm{CO})_{6}$ vapor $\left[\mathrm{W}(\mathrm{CO})_{6}\right.$ is a high vapor pressure solid]. When a $\mathrm{W}(\mathrm{CO})_{6}$ molecule strikes the hot ribbon, the reaction $\mathrm{W}(\mathrm{CO})_{6} \rightarrow \mathrm{W}$ $+6 \mathrm{CO}$ takes place and tungsten is deposited on the ribbon surface. The $\mathrm{CO}$ gas released in the process was pumped off every $10 \mathrm{~min}$. An hour of coating time gave us a coating thickness of about $2 \mu \mathrm{m}$. After the coating, a hard bake of $1600 \mathrm{~K}$ (yellow-white) for $1 \mathrm{~h}$ removed residual impurities from the surface. The same coating process was also applied to the molybdenum ribbon holder.

After installing the detector into the vacuum system in which our experiments are performed, a soft bake at a temperature of $1400 \mathrm{~K}$ (yellow hot) for several minutes cleaned the ribbon. A similar bake is necessary every time after exposing the detector to air. During these bakes a molybdenum 
heat shield attached to a linear motion feedthrough is positioned between the ribbon and the MCPs to protect the MCPs from the radiation heating by the ribbon. During operation of the detector, the ribbon is heated to around $1000 \mathrm{~K}$ (red hot). It is spring-loaded to prevent sagging when heated.

A copper heat shield houses the detector. The heat shield, and in turn the detector itself, is cooled by a copper braid connected to a liquid nitrogen trap. Before we added the cooling, we found that the MCPs were significantly heated by the ribbon. This was indicated by a reduction in the resistance of the MCPs when the ribbon was heated and, we believe, the premature, gradual, irreversible loss of gain in the MCPs. Also, we noticed that the background of the detector increased on a minutes time scale after the ribbon was turned on. This, we believe, was due to alkali atoms boiling off as neutrals from the glass of the MCPs as they heated up and then being detected at the ribbon. Cooling the detector solved these problems. In addition, because $\mathrm{Rb}$ has a relatively high vapor pressure ( $\sim 10^{-6}$ Torr) at room temperature, experiments sometimes have problems caused by backgrounds of atoms reemitted from various room temperature surfaces. The cold surfaces of the detector and its housing provide surfaces to trap those atoms and help reduce the overall system pressure.

\section{CHARACTERIZATION}

In this section we describe measurements that we carried out to characterize the detector. These measurements were not intended to calibrate the device but rather to check that it operates, generally, in the expected fashion.

\section{A. Efficiency}

A theoretical estimate for the quantum efficiency of the detector is given by

$$
\mathrm{QE}=P_{\text {ion }} P_{\text {pulse }} P_{\text {detect }} \text {. }
$$

Here, $P_{\text {ion }}$ is the probability that an atom incident on the hot ribbon is boiled off as a positively charged ion, $P_{\text {pulse }}$ is the probability that an ion will initiate an avalanche in the MCP, and $P_{\text {detect }}$ is the probability that the television system and image processing software will identify a given pulse of electrons hitting the phosphor screen as a count. Assuming $\mathrm{Rb}$ atoms hitting the ribbon come into thermodynamic equilibrium with the surface, the ratio of atoms emerging as ions to those coming out as neutrals ${ }^{8}$ is $\sim \exp [-e(I-\phi) / k T]$. Here, $e I=4.18 \mathrm{eV}$ is the ionization potential of $\mathrm{Rb}, e \phi$ $=4.55 \mathrm{eV}$ is the work function of tungsten, and $k T$ $=0.086 \mathrm{eV}$ for a ribbon temperature of $1000 \mathrm{~K}$. Thus $P_{\text {ion }}$ $\approx 1$. Straub et al. ${ }^{9}$ have recently investigated the absolute detection efficiency of a MCP operated in a fashion similar to ours. They found that $P_{\text {pulse }} \approx 0.58$ for $5.4 \mathrm{keV}$ ions of any singly charged ion species. They note that this is very nearly the fractional open area of the first MCP, 0.61, and that the difference may be understood as the result of ions that land within a channel but very near the surface of the plate. The secondary electrons for these ions are pulled out of the channel by the fringing field of the electric field accelerating the ions into the MCP and therefore do not initiate an avalanche.
Ions in our detector impact the MCPs with an energy of $\sim 4 \mathrm{keV}$ and for the purposes of our analysis, it is adequate and reasonable to expect that $P_{\text {pulse }}$ is on the order of $1 / 2$. Finally, we found that varying the MCP gain (i.e., voltage) has virtually no impact on the actual number of pulses that are counted in the detector. This means that most avalanche electron pulses at the phosphor screen are much larger than the threshold size needed for detection and therefore $P_{\text {detect }}$ must be very nearly unity. Combining these results leads us to predict that the absolute quantum efficiency QE of the detector for $\mathrm{Rb}$ atoms is on the order of $1 / 2$.

To check that the efficiency of our detector has the order of magnitude of the above theoretical prediction we carried out the following experiment. A sample of ${ }^{87} \mathrm{Rb}$ atoms was slowed in a Zeeman $\sigma^{-}$slowing source ${ }^{10}$ and captured in a magneto-optical trap (MOT). ${ }^{11}$ The atoms were then further cooled by gradient cooling molasses ${ }^{12}$ and finally dropped freely onto the detector located $40 \mathrm{~cm}$ below. The temperature of the dropped atom sample was estimated using a timeof-flight technique. ${ }^{13}$ This involved measuring the temporal pulse width of a fluorescence signal from the dropped atoms as they passed through a standing wave probe beam $24 \mathrm{~cm}$ below the trap. From the measured temperature, $13 \mu \mathrm{K}$, we find how much the atom sample spreads out at the detector (it is much broader than the ribbon, in all dimensions). The total number of dropped atoms was estimated from an absolute measurement of the fluorescence from the atom trap. We can estimate the number of atoms hitting the ribbon from these two measurements and compare with the number of atoms that are actually detected.

Figure 2 is a sequence of frames captured from the television camera showing the dropped atoms. Adding up all spots on frames (a)-(c) gives a (best estimate) measured absolute quantum efficiency of $\sim 50 \%$. There are large uncertainties $(\sim$ a factor of 2$)$ in determining the total number of dropped atoms based on the atom trap fluorescence. However, the accuracy suffices to show that the order of magnitude of the quantum efficiency is as expected.

\section{B. Background and time response}

A notable feature of our detector is the very low background count rate that we achieve. To measure the intrinsic background count rate of the detector we operated the device in an ion pumped ultrahigh vacuum: $P<10^{-9}$ Torr. Even after coating the ribbon, the dominant source of background depends on the ribbon temperature and is likely residual low ionization potential species boiling off as ions. We found a few "hot spots" on the ribbon that gave large backgrounds. However, these only constitute $<1 \%$ of the ribbon area and can be ignored in operation. Besides this, there is a trade-off that needs to be made in choosing a ribbon temperature: lower temperatures give lower backgrounds but degrade the time response of the device. We empirically chose to operate at a ribbon temperature of $T \approx 1000 \mathrm{~K}$. At this temperature, the background rate across the whole detector was $\sim 30 \mathrm{~Hz}$, and the time response was adequate. The frames in Fig. 2 were captured at a rate of $30 \mathrm{~Hz}$ and from them we can conclude that for the central $\sim 2 / 3$ of the ribbon, the time response of the detector is no worse than 1/30 s. 
(a)

(b)

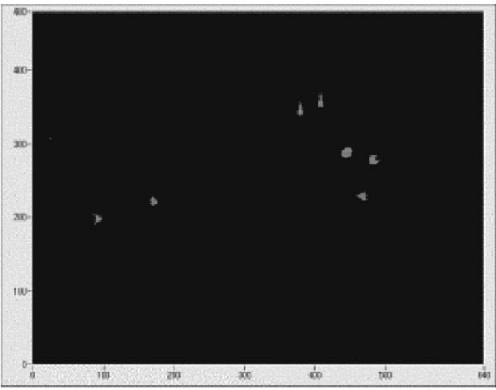

(c)
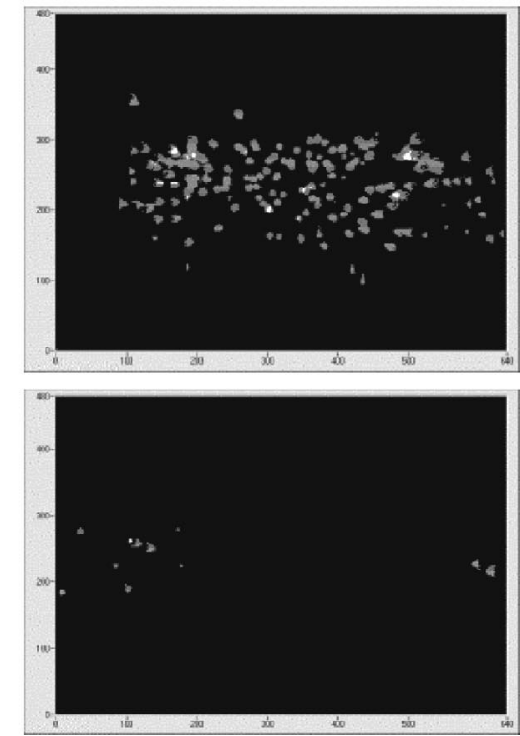

FIG. 2. Detection efficiency measurement. A measured number of Rb atoms in a magneto-optical trap (MOT) were dropped onto the detector. The atom temperature was measured to be $13 \mu \mathrm{K}$ and, thus, we can estimate the incident flux of atoms on the detector. Shown is a sequence of frames that were grabbed at a frequency of $30 \mathrm{~Hz}$ from the television camera that monitors the phosphor screen of the detector. The spots correspond to individual atoms arriving at the detector, and for quantitative imaging work we locate the center of each spot. By comparing the total number of spots in these frames with our estimate of the atom flux, we determine a detection efficiency of $\sim 50 \%$ ( \pm a factor of 2 ). By noting that most of the atoms arrive during a single frame [i.e., (b)], we can put an upper limit on the time constant of the system-essentially the time constant of the hot ribbon-of $1 / 30 \mathrm{~s}$. The ends of the ribbon are cooler and therefore have a longer response time, as can be seen in (a) and (c).

The particle counting capability of the detector suggests its utility for time-of-flight measurements or other low count rate experiments where timing information is important. For such applications we can theoretically estimate the time response of the detector. For ribbon temperatures between 1000 and $1400 \mathrm{~K}$ the "ribbon release time" will usually dominate the detector's time response. Note: for the present detector we restrict operation below $1400 \mathrm{~K}$ because of concerns about damaging the MCP. Hughes and Levinstein ${ }^{14}$ have measured release times for $\mathrm{Rb}$ on tungsten and found values that range from $\sim 10 \mathrm{~ms}$ at $1000 \mathrm{~K}$ down to $\sim 10 \mu \mathrm{s}$ at $1400 \mathrm{~K}$. In contrast, the ion travel time from the ribbon to the MCPs is $\sim 0.1 \mu$ s with a time-of-flight variation of $\lesssim 0.1$ $\mu$ s due to the ribbon tilt, the electron transit time through the MCPs and to the phosphor is $<1 \mathrm{~ns}$, and the phosphor decay time is $\sim 10 \mu \mathrm{s}$. Given the uncertainty of our estimates of the ribbon temperature, the expected $10 \mathrm{~ms}$ release time at $1000 \mathrm{~K}$ is consistent with the observations of Fig. 2. Moreover, in principle, at the expense of larger backgrounds, it

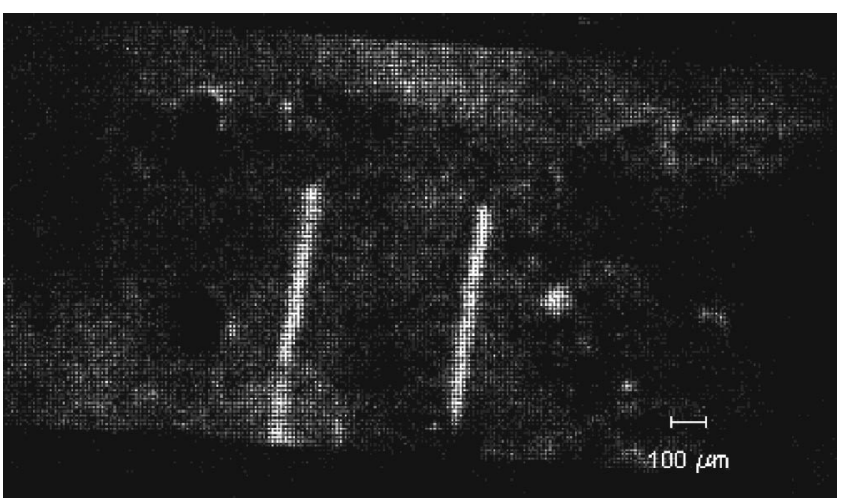

FIG. 3. Resolution measurement. Atoms from a distant point source impinge on a mask with two $15 \mu \mathrm{m}$ slots that are separated by $500 \mu \mathrm{m}$, and the transmitted atoms are intercepted by the detector. Shown is the output from the television camera of the detector. Quantitative analysis of the images grabbed from the television camera confirm that the spatial resolution of the detector is $\sim 20 \mu \mathrm{m}$ in the direction that is perpendicular to the slits. This is consistent with estimates in which the dominant effect that limits the resolution of the detector is the thermal distribution of the transverse velocities of the ions that emerge from the heated foil. Note: the artifacts seen in the picture were caused by damage to the MCPs as a result of high voltage arcing. This measurement was made in a different system than the previous one and the vacuum was considerably poorer.

should be possible to determine timings for the arrival of individual particles to within a couple of tens of $\mu$ s with suitable electronics.

\section{Resolution}

There are several features of the detector that degrade its ultimate resolution, ${ }^{15}$ including the pore diameter of the MCPs and the finite pixel size of the CCD camera. It turns out that the dominant resolution-limiting mechanism results from the thermal transverse velocities of the ions boiled off the hot ribbon. These velocities cause a spatial spread of impacts at the MCP. We numerically solved the equations of motion of an ion in the electric field between the ribbon and the MCP, and found that, at the center of the ribbon, $1000 \mathrm{~K}$ ions accelerated from a single point would produce a $50 \mu \mathrm{m}$ diameter spot on the MCP. As discussed above, the tilt of the detector spreads out displacements along the $y$ direction and therefore effectively improves the resolution in that dimension by a factor of 3.2. Thus we expected a resolution of about $16 \mu \mathrm{m}$. However, we had serious concerns that the resolution of the detector might be additionally very significantly degraded if atoms moved large distances along the hot ribbon before they were ionized.

To check on this and find the order of magnitude of the spatial resolution we carried out the following measurement. First, to make a (near) point source of atoms, we placed a $200 \mu \mathrm{m}$ diameter pinhole in front of a Rb oven. Next, we positioned a mask with two $15 \mu \mathrm{m}$ slits separated by $500 \mu \mathrm{m}$ in a plane $94 \mathrm{~cm}$ beyond the pinhole. The detector was located $15 \mathrm{~cm}$ further downstream. Figure 3 shows the image of the mask created by the atoms at the detector. A quantitative analysis of this and similar images gives a resolution of 15-20 $\mu \mathrm{m}$, which is consistent with our expectations and eliminates the concern that the atoms move large distances on the heated surface prior to ionization. 


\section{Future improvements}

Incremental improvements over the detector described in this work are possible. By increasing the voltage between the ribbon and the MCP or merely using that part of the ribbon nearer the MCP, it should be possible to improve the spatial resolution of the device by a factor of 2 or 3 . Deconihout et $a l .{ }^{16}$ have recently shown that moderate improvements in detection efficiency may be achieved by putting a grid in front of the MCP and biasing it so as to direct secondary electrons back into the MCP. A faster television camera could improve the response time of the detector. Finally, there is no fundamental limit to the length and width of the ribbon.

\section{ACKNOWLEDGMENTS}

The authors acknowledge technical assistance from Josh Holt, Tom Kelch, and the OSU machine shop, as well as Dedra Demaree, Reni Ayachitula, Rob Coridan, and Ralph Hutchinson. The authors also are grateful for helpful conversations with Nate Woodard. This research was supported by the Physics Division of the National Science Foundation.

\footnotetext{
${ }^{1}$ M. Morinaga, M. Yasuda, T. Kishimoto, F. Shimizu, J.-i. Fujita, and S. Matsui, Phys. Rev. Lett. 77, 802 (1996).

${ }^{2}$ F. Shimizu, Phys. Rev. Lett. 86, 987 (2001).

${ }^{3}$ R. Delhuille et al., Rev. Sci. Instrum. 73, 2249 (2002).
}

${ }^{4}$ J. B. Taylor, Phys. Rev. 35, 375 (1930).

${ }^{5}$ I. Langmuir and K. H. Kingdon, Proc. R. Soc. London, Ser. A 107, 61 (1925).

${ }^{6}$ B. Sheehy, S-Q. Shang, R. Watts, S. Hatamian, and H. Metcalf, J. Opt. Soc. Am. B 6, 2165 (1989).

${ }^{7}$ J. W. Frazer, R. P. Burns, and G. W. Barton, Rev. Sci. Instrum. 30, 370 (1959).

${ }^{8}$ N. F. Ramsey, Molecular Beams (Oxford University, New York, 1985), XIV 3.2.

${ }^{9}$ H. C. Straub, M. A. Mangan, B. G. Lindsay, K. A. Smith, and R. F. Stebbings, Rev. Sci. Instrum. 70, 4238 (1999).

${ }^{10}$ T. E. Barrett, S. W. Dapore-Schwartz, M. D. Ray, and G. P. Lafyatis, Phys. Rev. Lett. 67, 3483 (1991).

${ }^{11}$ D. W. Sesko, T. G. Walker, and C. E. Wieman, J. Opt. Soc. Am. B 8, 946 (1991).

${ }^{12}$ P. D. Lett, W. D. Phillips, S. L. Rolston, C. E. Tanner, R. N. Watts, and C. I. Westbrook, J. Opt. Soc. Am. B 6, 2084 (1989).

${ }^{13}$ C. G. Townsend, N. H. Edwards, C. J. Cooper, K. P. Zetie, and C. J. Foot, Phys. Rev. A 52, 1423 (1995).

${ }^{14}$ F. L. Hughes and H. Levinstein, Phys. Rev. 113, 1029 (1959).

${ }^{15}$ Because of the various resolution degrading mechanisms, the apparent size of a sample, measured from the image made by the detector, will always be blurred to somewhat larger than the actual size of the sample. In this article, we use "resolution" to mean, quantitatively, "the apparent width (FWHM), that would be measured for an infinitesimally narrow atomic beam at the detector input." Note: the "resolution" of the detector in the $x$ direction is different than in the $y$ direction: a beam that was infinitesimally narrow in both $x$ and $y$ would appear (e.g., in Fig. 3) to be $\sim 50 \times 20 \mu \mathrm{m}^{2}$

${ }^{16}$ B. Deconihout, F. Vurpillot, M. Bouet, and L. Renaud, Rev. Sci. Instrum. 73, 1734 (2002). 